\title{
Bacteriemia por Staphylococcus aureus con sensibilidad disminuida heterogénea a vancomicina: reporte de un caso
}

\author{
Staphylococcus aureus bacteremia with heterogeneous intermediate-level \\ vancomycin resistance: a case report
}

Sabina Lissarrague1 , Judith Bernstein², Juan Pablo Stagnaro², Celia Schell², Mónica Sparo 1,2*

\section{Resumen}

Staphylococcus aureus meticilina-resistente (SAMR) es una causa frecuente de bacteriemias intrahospitalarias. Para su tratamiento se utiliza vancomicina y han emergido cepas con sensibilidad disminuida heterogénea (h-VISA) que albergan subpoblaciones con sensibilidad reducida a vancomicina. Se comunica un caso de bacteriemia intra-tratamiento con vancomicina por SAMR h-VISA. El aislamiento muestra sensibilidad a vancomicina ( $\left.\mathrm{CIM}_{\mathrm{van}}: 1 \mu \mathrm{g} / \mathrm{mL}\right)$, sin embargo E-test GRD sugiere h-VISA (CIM $\mathrm{van}_{2}: 2 \mu \mathrm{g} / \mathrm{mL}$ y CIM tei $\left.: 8 \mu \mathrm{g} / \mathrm{mL}\right)$. El análisis del perfil poblacional - área bajo la curva (PAP-AUC) valida este hallazgo. Se rota a linezolid con resolución clínica.

Palabras clave: Staphylococcus aureus; bacteriemia; vancomicina; h-VISA; E-test GRD; perfil poblacional.

\section{Abstract}

Methicillin-resistant Staphylococcus aureus (SAMR) is a common cause of nosocomial bacteremia. Vancomycin, a glycopeptide, is widely employed for the therapy of SAMR infections. In recent years, heterogeneous vancomycin-intermediate strains (h-VISA) have emerged. We report a case of intra-treatment bacteremia caused by SAMR h-VISA. The isolate shows susceptibility to vancomycin (MIC $: 1 \mu \mathrm{g} /$ $\mathrm{mL}$ ). But the GRD E-test suggests $\mathrm{h}$-VISA ( $\mathrm{MIC}_{\mathrm{van}}: 2 \mu \mathrm{g} / \mathrm{mL}$ and $\mathrm{MIC}_{\mathrm{tei}}: 8 \mu \mathrm{g} / \mathrm{mL}$ ). The population analysis profile - area under the curve (PAP-AUC) validates SAMR h-VISA. Rotation of antibiotic therapy with linezolid is done, with good clinical outcome.

Keywords: Staphylococcus aureus; bacteremia; vancomycin; h-VISA; GRD E-test; population profile.

Fecha de envío: 2020-03-10 - Fecha de aceptación: 2020-12-15

\section{Introducción}

Staphylococcus aureus es uno de los principales agentes de bacteriemias de origen hospitalario y con una elevada morbi-mortalidad. Esta bacteria representa un desafío para la salud pública por su virulencia y habilidad para desarrollar resistencia antimicrobiana. Para el tratamiento de las infecciones por S. aureus meticilina-resistente (SAMR) se utiliza vancomicina. Se han descrito cepas con sensibilidad disminuida a vancomicina (VISA), con concentración inhibitoria mínima $\left(\mathrm{CIM}_{\mathrm{van}}\right): 4-8 \mu \mathrm{g} / \mathrm{mL}$ y cepas con sensibilidad disminuida heterogénea ( $\mathrm{h}$-VISA) que son sensibles según las pruebas habituales utilizadas por los laboratorios de microbiología clínica ( CIM $_{\text {van }}: 1-2 \mu \mathrm{g} / \mathrm{mL}$ ), pero que albergan subpoblaciones VISA de detección dificultosa (Amatya et al., 2014).
Se comunica un caso de bacteriemia, intra-tratamiento con vancomicina, por SAMR fenotipo h-VISA.

\section{Caso clínico}

En marzo de 2019 ingresa al Hospital Ramón Santamarina del Municipio de Tandil, Provincia de Buenos Aires, Argentina (HRS) paciente, género masculino de 73 años con antecedentes de hipotiroidismo, cardiopatía isquémica, hipertensión arterial, fibrosis pulmonar y enfermedad pulmonar obstructiva crónica (EPOC). Presenta un cuadro de neumonía complicada con lesiones cavitadas compatibles con abscesos pulmonares (según estudios complementarios de TAC). Se realiza ecocardiograma que no presenta patología. Se solicita hemocultivos ( 2 muestras, sistema automatizado BACT

(1) Laboratorio Microbiología Clínica, Hospital Municipal Ramón Santamarina, Sistema Integrado de Salud del Municipio de Tandil, Provincia de Buenos Aires, Argentina

(2) Microbiología y Parasitología (CUDEMyP), Facultad Ciencias Médicas, UNLP, Hospital Municipal Ramón Santamarina. Buenos Aires, Argentina

*Autor de correspondencia: monicasparo@gmail.com 
ALERT $^{\oplus}$ 3D, bioMèrieux, Argentina). A las $24 \mathrm{~h}$ el Laboratorio de Microbiología Clínica del HRS aísla en las 2 muestras de hemocultivo SAMR (prueba de aglutinación de látex para PLP2a: positiva) sensible a vancomicina según criterio del Clinical and Laboratory Standards Institute (CLSI, 2019) con CIM $_{\text {van }}: 1,0 \mu \mathrm{g} / \mathrm{mL}$ (sistema VITEK ${ }^{\circledast}$ $2 \mathrm{C}$, bioMèrieux, Argentina). Se le coloca un catéter venoso central (CVC) y se inicia tratamiento con vancomicina (dosis de carga y mantenimiento ajustadas al peso corporal del paciente). A los 12 días de tratamiento, el paciente se encuentra afebril y lúcido, con persistencia de lesiones cavitadas pulmonares; se efectúa hemocultivo de control (2 muestras) que resulta negativo. Se continúa tratamiento con vancomicina. Intra-tratamiento, (20 días con antibiótico, niveles séricos de vancomicina (valle, 4 dosajes):15,8-18,1 $\mu \mathrm{g} / \mathrm{mL}$ ) el paciente presenta fiebre y escalofríos. Se extrae el CVC y se toman 2 muestras de hemocultivo. Se recupera en una de dos muestras de hemocultivo y del cultivo de la punta del CVC (> 15 UFC; según técnica semi-cuantitativa de Maki, 1977) SAMR con $\mathrm{CIM}_{\text {van}}$ : $1,0 \mu \mathrm{g} / \mathrm{mL}$ (VITEK 2C). Para la detección de h-VISA se efectúa como prueba de tamizaje E-test GRD (Liofilchem ${ }^{\circledR}$, Italia) siguiendo las instrucciones del fabricante; obteniendo $\mathrm{CIM}_{\text {van }}: 2 \mu \mathrm{g} / \mathrm{mL}$ y CIM $8 \mu \mathrm{g} / \mathrm{mL}$ (E-test GRD: h-VISA CIM $\geq 8 \mu \mathrm{g} / \mathrm{mL}$ para vancomicina o teicoplanina). También se realiza el análisis del perfil poblacional (PAP) con el análisis del área bajo la curva (AUC) del aislamiento en estudio, de S. aureus ATCC 700698 (h-VISA) y de S. aureus ATCC 25923, sensible a vancomicina (Satola et al., 2011). Se observa un cociente AUC cepa en estudio/S. aureus ATCC 700698=0,97 (h-VISA: cociente $A U C=0,90-1,3$ ) comprobando la emergencia de una cepa h-VISA en el paciente (Figura 1). El rango de las concentraciones de vancomicina utilizadas en el PAP-AUC es 0-6 $\mu \mathrm{g} / \mathrm{mL}$.

Se rota el antimicrobiano, iniciando tratamiento con linezolid ( $\mathrm{CIM}_{\mathrm{LZD}}$ : $2 \mu \mathrm{g} / \mathrm{mL}$, VITEK 2C). A los 30 días posteriores a su internación el paciente egresa del HRS, afebril, estable pero con persistencia de lesiones cavitadas; continuando el tratamiento de modo ambulatorio con posterior resolución clínica de la infección.

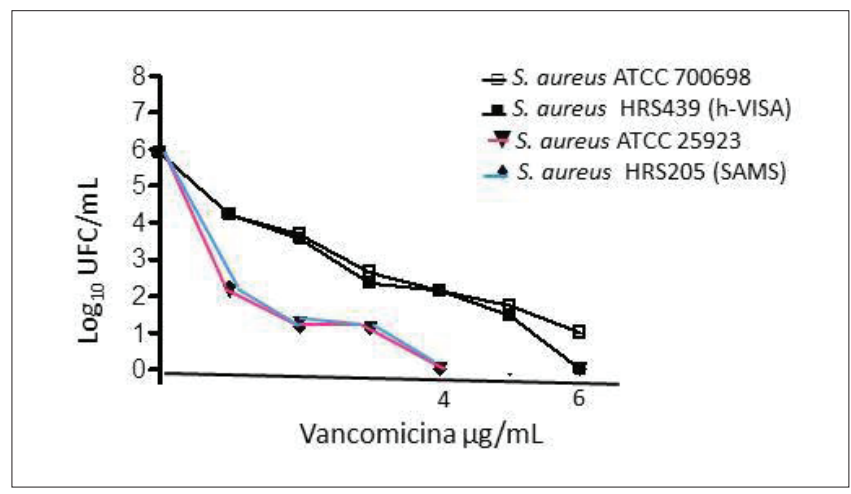

Figura 1: $\log _{10} \mathrm{UFC} / \mathrm{mL}$ vs $\mathrm{CIM}_{\mathrm{van}} \mu \mathrm{g} / \mathrm{mL}$. Análisis del perfil poblacional con análisis del área bajo la curva (PAP-AUC). GraphPad Prism 8.0 (GraphPad Prism Software Inc., San Diego, CA).

\section{Discusión}

A nivel mundial, SAMR continúa siendo uno de los patógenos más comunes de la comunidad y de infecciones asociadas a los cuidados de la salud. Vancomicina es el fármaco de primera línea para las infecciones por SAMR. El mayor uso de este glucopéptido ha inducido la emergencia de cepas VISA y h-VISA. Debido a la dificultad en la detección de los aislamientos clínicos h-VISA, su prevalencia e impacto clínico no se han estudiado adecuadamente. En contraste con la baja frecuencia de la resistencia a vancomicina (SAVR), en las cepas de SAMR se ha incrementado el reporte de fallas terapéuticas con esta droga. La disminución en la eficacia del tratamiento con vancomicina se ha descrito en infecciones producidas por cepas VISA y h-VISA; aunque no se ha observado incremento en la mortalidad (Wang et al., 2013).

Las cepas h-VISA están diseminadas a nivel mundial, presentando una frecuencia variable entre los aislamientos clínicos de SAMR. En un meta-análisis realizado por Van Hal \& Paterson (2011), la tasa global de h-VISA en Europa entre las cepas de SAMR fue de 1,3\%. En una evaluación de 22 años en EE.UU. se observó un incremento de h-VISA de 2,2\% a 8,3\% (Rybak et al., 2008). En Brasil, Da Costa (2016) en un estudio realizado durante el periodo 2011-2013 en un hospital universitario de Río de Janeiro detectó 1 aislamiento h-VISA sobre 31 SAMR analizados.

En Chile, el primer aislamiento de h-VISA se comunicó en el año 2015 en una paciente del Hospital Clínico Regional de Concepción que había recibido tratamiento previo con vancomicina (Vega et al., 2015).

En Argentina, Errecalde (2015) reportó un aislamiento clínico de VISA, sin embargo el presente caso clínico es el primero documentado de bacteriemia producida por h-VISA.

La evolución clínica de la infección por h-VISA varía con el sitio y la gravedad del cuadro clínico y con la presencia de algún cuerpo extraño. Se encontró que los marcadores de una potencial infección por h-VISA son la presencia de elevada carga bacteriana, fiebre persistente y bacteriemia, empeoramiento de los signos de infección luego del comienzo de la terapia con vancomicina y presencia de dispositivos protésicos como implantes ortopédicos, válvulas artificiales y marcapasos (Amatya et al., 2014). Es importante destacar que la disminución de la acción de vancomicina en h-VISA / VISA también puede ser atribuida a otras características biológicas de la bacteria asociadas a la mutación de los genes reguladores (vraSR, gra $R S$, walk $R K$ ) y el gen $r p o B$, ocasionando una disminución en la velocidad de crecimiento y en la actividad autolítica y un engrosamiento de la pared celular con reducción de entrecruzamiento del peptidoglicano. 
La actividad bactericida de vancomicina se ve también comprometida por la formación de biopelículas, como sucede en las infecciones crónicas y asociadas a dispositivos médicos. Se ha observado que aislamientos de SAMR con $\mathrm{CIM}_{\text {van }}: 1-2 \mu \mathrm{g} / \mathrm{mL}$ tienen mayor asociación con fracaso terapéutico con este fármaco, ya que la infección puede ser ocasionada por cepas h-VISA / VISA (Wang et al., 2013; Wang et al., 2017).

En un aislamiento clínico de SAMR, si la proporción de una población no sensible a vancomicina es suficientemente alta, se puede detectar con las pruebas estándar de resistencia antimicrobiana. Sin embargo, en las cepas h-VISA las subpoblaciones ocurren a una frecuencia muy por debajo del umbral del inóculo estándar y pueden interpretarse como sensibles con las pruebas habituales de resistencia antimicrobiana del laboratorio de microbiología clínica. En las cepas h-VISA se ha encontrado que la mayoría de la población presenta un rango de $\mathrm{CIM}_{\mathrm{van}} \leq 2 \mu \mathrm{g} / \mathrm{mL}$, aunque contiene una subpoblación de células resistentes con una frecuencia aproximada de 1 en $10^{5}$ células- $10^{6}$ células (Plipat et al., 2005). La detección de h-VISA es un desafío para los laboratorios de microbiología clínica pues el método fenotípico confiable (estudio del perfil poblacional) no está disponible para la práctica de rutina de laboratorio, siendo su realización tediosa y compleja. Una prueba sugerente de h-VISA es E-test GRD que utiliza tiras con doble cara conteniendo concentraciones crecientes de vancomicina y teicoplanina en lados opuestos en un medio de cultivo con suplemento nutricional (Riederer et al., 2011). Sin embargo, según distintos autores, los aislamientos de SAMR identificados por E-test GRD deben confirmarse mediante el análisis del perfil poblacional con el análisis debajo de la curva (PAP-AUC). Este método es considerado como "estándar de oro" para la detección de h-VISA; consiste en evaluar un aislamiento a concentraciones crecientes de antibiótico y diferentes diluciones de inóculo bacteriano, realizando conteo de bacterias viables. Finalmente se grafican los resultados del número de UFC, frente a la concentración de vancomicina y se realiza un cálculo de la proporción del valor obtenido de AUC, entre el aislamiento en evaluación contra la cepa de referencia (Mu3). Se interpreta como $S$. aureus sensible a vancomicina (VSSA), PAP-AUC $<0,9$; h-VISA, PAP-AUC=0,9-1,3 y VISA, PAP-AUC > 1,3 (Howden et al., 2010; Satola et al., 2011; Huang et al., 2016). El aislamiento h-VISA del presente caso clínico no fue detectado a través de las pruebas de resistencia realizadas por el sistema automatizado (Vitek 2C), sin embargo las pruebas E-test GRD y PAP-AUC fueron instrumentos útiles para su pesquisa.

\section{Conclusiones}

La exposición prolongada a vancomicina en los pacientes puede contribuir a la generación de cepas h-VISA, originando fallas en el tratamiento con este fármaco. El reporte del presente caso pretende alertar sobre la emergencia de cepas h-VISA en pacientes con bacteriemia por SAMR y terapia prolongada con vancomicina. En virtud de que las pruebas habituales de resistencia antimicrobiana no detectan los aislamientos h-VISA, este reporte muestra la utilidad de otros métodos fenotípicos como alternativas factibles para su pesquisa.

\section{Aspectos éticos}

El presente caso fue desarrollado en el contexto de la ejecución del Proyecto de Investigación (Código M201) del Programa de Incentivos Docentes-Investigadores de la Secretaría de Ciencia y Técnica de la Universidad Nacional de La Plata (SECyT-UNLP, Argentina) que ha sido aprobado (Protocolo 21/2016) por el "Comité de Bioética de la Facultad de Ciencias Médicas de la Plata" (COBIMED).

\section{Reconocimientos}

El presente trabajo de investigación ha sido realizado en el marco del proyecto del Programa de Incentivos Docentes-Investigadores, SECyT-UNLP, Argentina.

Los autores declaran no tener conflictos de intereses.

\section{Referencias}

Amatya R, Devkota P \& Gautam A. (2014). Reduced susceptibility to vancomycin in methicillin resistant Staphylococcus aureus: a time for action. Nepal Med Coll J 16, 42-44.

Clinical and Laboratory Standards Institute (2019). Performance Standards for Antimicrobial Susceptibility Testing, 30th Edition. Document M100. Clinical and Laboratory Standards Institute,Wayne, PA.9.

Da Costa TM, Morgado PG, Cavalcante FS, Damasco AP, Nouér SA \& Dos Santos KR. (2016). Clinical and Microbiological Characteristics of Heteroresistant and Vancomycin-Intermediate Staphylococcus aureus from Bloodstream Infections in a Brazilian Teaching Hospital. PLoS One 8, 1-11.

Errecalde L, Ceriana P, Gagetti P, Erbin M, Duarte A, Rolón M, et al. (2013). First isolation in Argentina of community-acquired methicillin-resistant Staphylococcus aureus with intermediate susceptibility to vancomycin and nonsusceptibility to daptomycin. Revista Argentina de Microbiolgía 45, 99-103.

Howden BP, Davies JK, Johnson PD, Stinear TP \& Grayson ML. (2010). Reduced vancomycin susceptibility in Staphylococcus aureus, including vancomycin-intermediate and heterogeneous vancomycin-intermediate strains: resistance mechanisms, laboratory detection, and clinical implications. Clinical Microbiology Reviews 23, 99-139. 
Huang SH, Chen YC, Chuang YC, Chiu SK, Fung CP, Lu PL, et al. (2016). Prevalence of vancomycin-intermediate Staphylococcus aureus (VISA) and heterogeneous VISA among methicillin-resistant $S$. aureus with high vancomycin minimal inhibitory concentrations in Taiwan: A multicenter surveillance study, 2012-2013. Journal of Microbiology Immunology and Infection 49, 701-707.

Maki DG, Weise CE \& Sarafin HW. (1977). A semiquantitative culture method for identifying intravenous-catheter-related infection. The New England Journal of Medicine 296, 1305-1309.

Plipat N, Livni G, Bertram H \& Thomson RB Jr. (2005). Unstable vancomycin heteroresistance is common among clinical isolates of methiciliin-resistant Staphylococcus aureus. Journal of Clinical Microbiology 43, 2494-2496.

Riederer K, Shemes S, Chase P, Musta A, Mar A \& Khatib R. (2011). Detection of intermediately vancomycin-susceptible and heterogeneous Staphylococcus aureus isolates: comparison of E-test and Agar screening methods. Journal of Clinical Microbiology 49, 2147-2150.

Rybak MJ, Leonard SN, Rossi KL, Cheung CM, Sadar HS \& Jones RN. (2008). Characterization of vancomycin-heteroresistant Staphylococcus aureus from the metropolitan area of Detroit, Michigan, over a 22-year period (1986 to 2007). Journal of Clinical Microbiology

46, 2950-2954.
Satola SW, Farley MM, Anderson KF \& Patel JB. (2011). Comparison of detection methods for heteroresistant vancomycin-intermediate Staphylococcus aureus with the population analysis profile method as the reference method. Journal of Clinical Microbiology 49, 177-183.

Van Hal SJ \& Paterson DL. (2011). Systematic review and metaanalysis of the significance of heterogeneous vancomycin- intermediate Staphylococcus aureus isolates. Antimicrobial Agents of Chemotherapy 55, 405-410.

Vega F, Alarcón P, Domínguez M, Bello H, Riedel G, Mella S, et al. (2015). Aislamiento de Staphylococcus aureus hetero-resistente a vancomicina en Hospital Clínico Regional de Concepción, Chile. Revista Chilena de Infectología 32, 588-590.

Wang Y, Hu YJ, Ai XM, Xu HT \& Sun TY. (2013). Prevalence and clinical prognosis of heteroresistant vancomycin-intermediate Staphylococcus aureus in a tertiary care center in China. Chinese Medical Journal 126, 505-509.

Wang Y, Li X, Jiang L, Han W, Xie X, Jin Y, et al. (2017). Novel Mutation Sites in the Development of Vancomycin- Intermediate Resistance in Staphylococcus aureus. Frontiers in Microbiology 7, 1-12. 